\title{
NUMERICAL SIMULATION AND OPTIMIZATION OF HIGH PERFORMANCE SUPERSONIC NOZZLE AT DIFFERENT CONICAL
}

\section{ANGLES}

\author{
A.Shanthi Swaroopini ${ }^{1}$, M.Ganesh Kumar ${ }^{2}$, T.Naveen Kumar ${ }^{3}$ \\ ${ }^{1}$ M.Tech Thermal Engineering, Sanketika Institute of Technology \& Management, Vizag. \\ ${ }^{2}$ Asst. Professor, Dept of Mechanical Engineering, Sanketika Institute of Technology \& Management, Vizag. \\ ${ }^{3}$ Technical Assistant, Dept of Mechanical Engineering, Gayatri Vidya Parishad College of Engineering, Vizag.
}

\begin{abstract}
The Principle purpose of a nozzle is to accelerate the flow to higher exit velocities. The fluid acceleration is based on the design criteria and characteristics. To achieve good performance characteristics with minimum energy losses a nozzle must satisfy all the design requirements at all operating conditions. This is possible only when the nozzle theory is assumed to be isentropic irrespective of the changes in pressure, temperature and density which is generally caused due to formation of a Shock Wave. The thesis focuses on the design, development and optimization of a Supersonic Convergent-Divergent Nozzle where the analytical results are validated using theory calculations. The simulation work is carried out for CD Nozzles with different angles of divergence keeping the other inputs fixed. The objective of the proposed thesis is to show the best Expansion ratio, Nozzle Pressure ratio (NPR) and Nozzle Area Ratio(NAR) where the thrust obtained by the supersonic nozzle is maximum. The simulation is then repeated for expansion gas the results of which are later compared with standard air to show which possesses better performance characteristics. The Nozzle design chosen is based upon existing literature studies.
\end{abstract}

Key Words: CD Nozzle, Expansion Ratio, Nozzle Pressure Ratio (NPR), Nozzle Area Ratio(NAR),Divergence Angle etc...

\section{INTRODUCTION}

A Nozzle is one of the major ducts which is used to accelerate flow from a series of mach regimes. These mach regimes constitute to be Subsonic, Sonic, Supersonic \& Hypersonic regimes. The type of nozzle that is chosen for a particular application mainly depends on the exit velocity of the flow through nozzle and the design and operating conditions. The efficiency of a nozzle greatly effects the overall performance of the system where it is employed. The main applications of the nozzle covers majority of the engineering, chemical and medical fields. However we are mostly bothered about the design and optimization of the duct thus resulting in improving the overall efficiency and minimizing the work input to the system. Nozzles are mainly used to control the speed and rate of the flow along with the direction and the pressure of the flow stream that emerges from them.

\subsection{Literature Review}

Convergent Divergent Nozzles are mostly used for Rockets, Jets and High speed Missiles which operate at relatively high mach speeds ranging from mach 2 to mach 5 . The purpose of the $\mathrm{CD}$ nozzle is to convert thermal or heat energy to kinetic energy to obtain a very high speed exhaust which in turn gives an equal amount of thrust as per Newton's Third Law of motion. Nozzle shapes play a significant role in its performance and requires an in deep study to design for a specific use or application.
The Convergent part of the nozzle is generally designed in such a way that it receives maximum amount of fluid mass flow and so it is designed to operate for bigger volumes. The size and dimensions of the divergent part plays a very important role in deciding the nozzle performance. Some parameters that best describe the performance characteristics of a CD nozzle are Nozzle Pressure Ratio (NPR), Nozzle Area Ratio (NAR) or Expansion Ratio and the Divergence angle. The changes in any of these parameters will greatly affect the nozzle performance depending on its design.

G.Satyanarayana, Ch.Varun and S.S.Naidu ${ }^{[1]}$ have carried out the analysis of flow within convergent-divergent supersonic nozzle for different cross sections like rectangular, square and circular by using ANSYS FLUENT 12.0. They performed the analysis depending on the shape of the supersonic nozzle keeping the same input conditions. They used the application of a nozzle as torpedo and carried out the work for best suited nozzle among those which are considered. Results have been shown for pressure, velocity and temperature variations for different supersonic nozzles and they found that rectangular nozzle gives high exit velocity compared to circular and square nozzles and they also mentioned that fluid properties are largely dependent on the cross section of the nozzle which greatly affects the fluid flow.

Omid Joneydi Shariatzadeh, Afshin Abrishamkar, and AliakbarJoneidi jafari ${ }^{[2]}$ have carried out a simulation 
analysis on a convergent divergent nozzle which is used in supersonic wind tunnels. They made use of software tools like Ansys Fluent \& Gambit to carry out the simulation analysis. They carried out the simulation on a $2 \mathrm{D}$ domain platform and made a numerical comparison between CFD modeling results and available measured data. They adopted two different models namely $\mathrm{K}-\mathcal{E}$ model and $\mathrm{K}-\omega$ model and noticed that $\mathrm{k}-\mathcal{E}$ model receives higher average values of Mach no in contrast to k- $\omega$ model. They observed that the computational values are not exactly matching with measured data and concluded by saying that the deviation is due to material property variation, slip factor \& wall friction. Finally they ended it by showing that the true result obtained in one scheme may differ with that of other and it would be a spitting image of the previous scheme.

Fred W.Steffen, H.George Krull and Ralph F.Schmiedlin ${ }^{[3]}$ have conducted tests on various $\mathrm{CD}$ nozzles by changing the divergence angles from $7^{\circ} 10^{\prime}$ to $50^{\circ}$ and nozzle area ratios from 1.39 to 3.81 over a wide range of nozzle pressure ratios to determine the effects of changing the divergence angle on the nozzles internal performance. They observed that the thrust coefficient decreased from 0.973 at a divergence angle of $7^{\circ}$ to 0.93 for a divergence angle of $50^{\circ}$. The also observed that the pressure ratios where separation occurred an increase in divergence angle effected the nozzle performance to a great extent. They finally concluded by saying that for a constant pressure ratio, the change in nozzle area ratio has a negligle effect on the performance of a nozzle.

\subsection{Problem Overview and Approach}

A De-laval nozzle is the one which is used in rocket motors that operate at supersonic to hypersonic mach speeds. The overall efficiency of the nozzle is affected by the length of the diverging part and the angle of divergence. A suitable angle of divergence and corresponding expansion ratio of the section is chosen that can operate at a given input conditions. Inorder to obtain the maximum possible efficiency of the nozzle, the same simulation analysis is carried out for different angles of divergence and the results so calculated from theoretical approach are validated with analytic results. This is done by changing the divergence angles and expansion ratios. The variation in the flow parameters and characteristics for different divergence angles are plotted accordingly.

Next phase of problem approach deals with the type of turbulence modeling method that is applied to the simulation analysis. The literature work shows that multiple modeling techniques have been employed to solve the same problem and the data gathered from them is used to compare the results obtained from theoretical analysis. Based on the results obtained for different modeling methods, the most appropriate results are found for the $\mathrm{K}$-epsilon turbulence modeling method. So our analysis work is carried out by employing the same method for all the nozzle designs.

\subsection{Objective of the Work}

The thesis focuses on the simulation work that is being carried out by employing a suitable turbulence modeling method giving out appropriate results which are later correlated with theoretically obtained results and those of existing literature studies. The objective is to show that the experimental results so obtained are found to be in good agreement with theoretically calculated data.

Inorder to achieve this the design requirements must be analyzed to the core extent and the construction features play a vital role in the output obtained. Once this is attained, the flow visualization inside the nozzle along with pressure, velocity, temperature and mach contours at the throat and exit of the nozzle are studied.

\section{Modeling}

The modeling and design of CD Nozzle is done using CATIA v5 R20. The CFD Solver chosen to carryout the Analysis work is ANSYS CFX. CFD Analysis is broadly used to carryout the simulation works involving fluid flows. This makes use of algorithms and numerical methodologies to solve for various aerodynamic, marine and fluid flow problems.

Draw the outer half section boundary of convergentdivergent nozzle with the dimensions specified in Table 1. Make sure the sketch is completely closed and dimensions are accurate while drawing. The closed sketch is now rotated about the central axis by using shaft command to obtain a complete nozzle surface.

Table 1: Dimensions of the Convergent - Divergent Nozzle

\begin{tabular}{|l|l|l|}
\hline S.no & Specifications & Dimensions (in) \\
\hline 1 & Total length & 25.913 \\
\hline 2 & Inlet radius & 6.5 \\
\hline 3 & Convergent length & 7.452 \\
\hline 4 & Throat radius & 3.054 \\
\hline 5 & Exit radius & 4.94 \\
\hline 6 & Divergent angle & 11 degrees \\
\hline
\end{tabular}

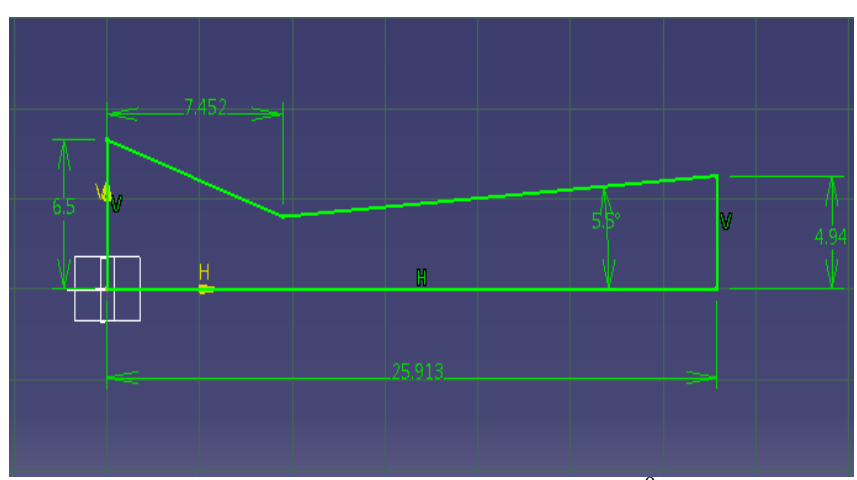

Fig-1:Sectional view of the nozzle at $11^{0}$ angle of divergence 
Similar procedure is repeated for modeling the nozzles of different conical or divergence angles. The length of the nozzle is fixed and the exit diameters are changed to model a nozzle with corresponding divergence angle. Table 2 shows the exit diameters and expansion ratios for respective divergence angles.

Table 2: Exit Radii \& Expansion ratios for different Divergence angles

\begin{tabular}{|c|c|c|}
\hline $\begin{array}{c}\text { Divergence } \\
\text { Angle }\end{array}$ & Exit Radius (in) & $\begin{array}{c}\text { Expansion Ratio or } \\
\text { Nozzle Area Ratio } \\
\text { (NAR) }\end{array}$ \\
\hline 5 & 3.831 & 1.5342 \\
\hline 9 & 4.481 & 2.0262 \\
\hline 11 & 4.94 & 2.4026 \\
\hline 15 & 5.445 & 2.84 \\
\hline 20 & 6.28 & 3.6172 \\
\hline
\end{tabular}

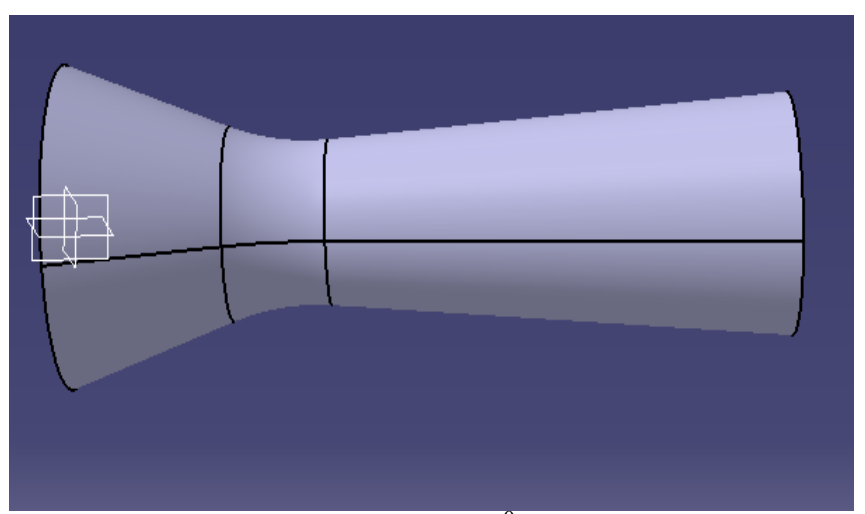

Fig-2: Generated Nozzle for $11^{0}$ angle of divergence

\section{ANALYSIS}

The tool used to carryout the CFD analysis is ANSYS 15.0 and the solver chosen is CFX. The Continuity, Momentum and Energy equations are the principle equations or laws used in CFD to solve for the required solution. Reynolds Averaged Navier Strokes(RANS) equations are generally used to find the solution theoretically. But for real fluid flow problems, a numerical method must be used where the algebraic approximations replace the RANS equations.

The ANSYS CFX tool is used to discretize the spatial domain using a mesh. A several set of finite volumes are constructed which are subjected to Gauss Divergence theorem which converts volume integrals comprising gradient operators and divergence to surface integrals. Many discretization methods developed are based on continuous functions of series expansions such as Taylor's series. The approximation accuracy is a function of mesh spacing and the time step factor of higher order terms in the series expansion. ANSYS CFX uses shape functions and second order approximations where the variations in each element such as tetrahedral, hexahedral, pyramid and wedge are described by tri-linear shape functions.
The nozzle modeled in Catia is imported to Ansys Workbench 15.0 where the solver chosen is Ansys CFX. There is no necessity of further domain construction of geometry in Ansys as the nozzle flow simulation is an internal flow type. After importing and updating the nozzle in geometry meshing is done by giving the details and sizing inputs as shown in Table 3.

Table -3: Details and Sizing inputs of a mesh

\begin{tabular}{|l|l|}
\hline Physics Preference & CFD \\
\hline Solver Preference & CFX \\
\hline Advanced size function & On: Proximity \& Curvature \\
\hline Relevance center & Fine \\
\hline Smoothing & High \\
\hline Maximum element size & $1.1781 \mathrm{e}-004 \mathrm{~m}$ \\
\hline Growth rate & 1.850 \\
\hline
\end{tabular}

The meshing is done for the given inputs and directed towards setup module where the named selection is given for each face and the type of heat transfer option and turbulence model is chosen. The heat transfer option is chosen as thermal energy and turbulence model as $\mathrm{k}-\mathcal{E}$ model. Give the input flow speed as $186 \mathrm{~m} / \mathrm{s}$ (Mach 0.2) in the default domain tab.

Next is the assigning of boundary conditions for each named selection and respective inputs given are shown in Tables 4,5 and 6.

Table-4: Boundary condition inputs for nozzle inlet

\begin{tabular}{|l|l|}
\hline Flow regime & Subsonic \\
\hline Mass and Momentum & Static Pressure as 80 bar \\
\hline Flow direction & Normal to boundary condition \\
\hline Turbulence option & Low Intensity $(=1 \%)$ \\
\hline Static Temperature & $1583 \mathrm{~K}$ \\
\hline
\end{tabular}

Table-5: Boundary condition inputs for nozzle outlet

\begin{tabular}{|l|l|}
\hline Flow regime & Subsonic \\
\hline Mass and Momentum & Static Pressure as 12.987 bar \\
\hline
\end{tabular}

Table-6: Boundary condition inputs for nozzle wall/surface

\begin{tabular}{|l|l|}
\hline Mass and Momentum & No Slip Wall \\
\hline Wall Roughness & Smooth \\
\hline Heat Transfer & Adiabatic \\
\hline
\end{tabular}

Now edit the basic settings in Solver control by changing the inputs accordingly as shown in table 7.

Table-7: Solver Control Settings For Solutions

\begin{tabular}{|l|l|}
\hline \multicolumn{2}{|l|}{ Flow Analysis } \\
\hline Advection Scheme & Upwind \\
\hline Turbulence Numeric & High resolution \\
\hline Convergence Control \\
\hline Minimum Iterations & 1 \\
\hline Maximum Iterations & 100 \\
\hline Convergence Criteria \\
\hline Residual type & RMS \\
\hline Residual target & $1 \mathrm{E}-04$ \\
\hline
\end{tabular}


Now the material input and properties are given depending on the type of fluid used to expand in the nozzle. The gases used in our analysis are air and expansion gas the properties of which are given as inputs as shown in tables 8 and 9.

Table-8: Material input and Properties for Air

\begin{tabular}{|l|l|}
\hline \multicolumn{2}{|l|}{ Thermodynamic Properties } \\
\hline Equation of State & Ideal gas (Air) \\
\hline Molar mass & $28.96 \mathrm{Kgk} / \mathrm{mol}$ \\
\hline Specific heat Capacity & $1004.4 \mathrm{~J} / \mathrm{KgK}$ \\
\hline Transport Properties \\
\hline Dynamic Viscosity & $1.831 \mathrm{e}-05 \mathrm{Kg} / \mathrm{ms}$ \\
\hline Thermal Conductivity & $0.0261 \mathrm{~W} / \mathrm{mK}$ \\
\hline
\end{tabular}

Table-9: Material input and Properties for Expansion Gas Thermodynamic Properties

\begin{tabular}{|l|l|}
\hline Equation of State & Ideal gas \\
\hline Molar mass & $23.05 \mathrm{Kgk} / \mathrm{mol}$ \\
\hline Specific heat Capacity & $2034.6 \mathrm{~J} / \mathrm{KgK}$ \\
\hline Transport Properties \\
\hline Dynamic Viscosity & $6.07 \mathrm{e}-05 \mathrm{Kg} / \mathrm{ms}$ \\
\hline Thermal Conductivity & $0.0706 \mathrm{~W} / \mathrm{mK}$ \\
\hline
\end{tabular}

The Solution running is started for the given inputs followed by iterations and wait till a pop up windows appears saying Results are normally completed. The next is the Postprocessing stage where the results are taken from the contour plots so obtained as shown in the following figures.

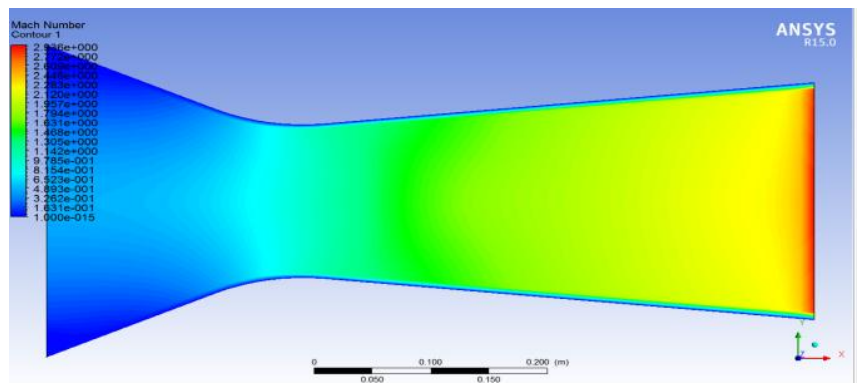

Fig-3: Mach Contours for $11^{0}$ divergence angle

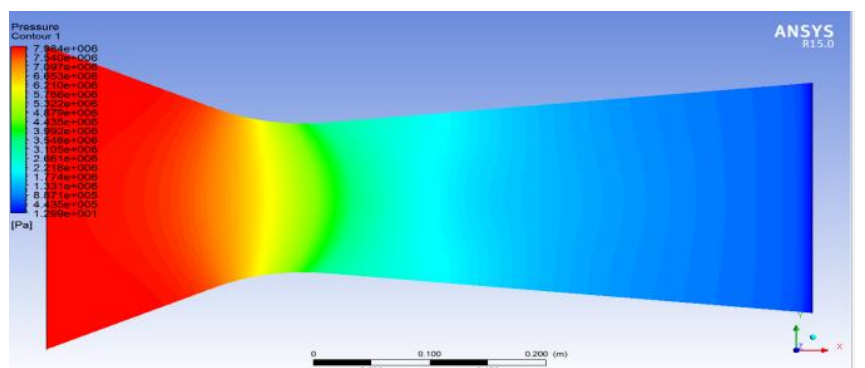

Fig-4: Pressure Contours for $11^{0}$ divergence angle

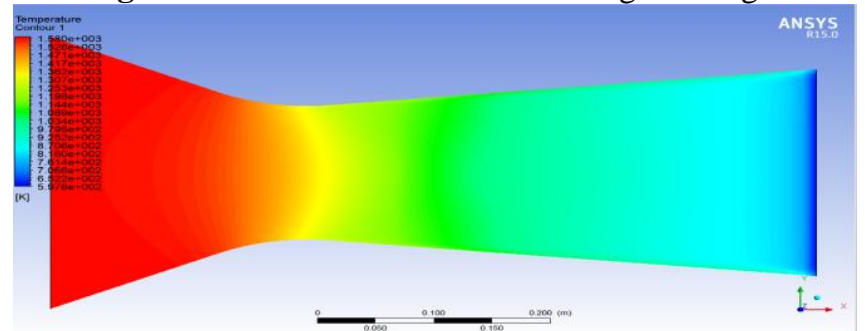

Fig-5: Temperature Contours for $11^{0}$ divergence angle
As per the Nozzle theory CD Nozzles are used for accelerating flows upto supersonic speeds. To obtain so the designed nozzle should be free from overexpansion and underexpansion shocks that generally originate with change in flow speed or change in nozzle pressure ratio. According to Nozzle theory, the gas velocity will increase upto sonic mach at the throat portion and to supersonic exit velocities at the outlet. The obtained Velocity, Pressure and Temperature contours follow the same strategy stating that obtained contour plots are in accordance with nozzle theory.

The same procedure is repeated to carryout analysis for remaining angles of divergence and results so obtained are compared with theoretical calculations.

\section{Theoretical Calculations of C-D Nozzle}

Properties and Inputs for Air:

Inlet Pressure $\left(\mathrm{P}_{\mathrm{o}}\right) \quad=80 \mathrm{bar}$

Inlet Temperature $\left(\mathrm{T}_{\mathrm{o}}\right) \quad=1583 \mathrm{~K}$

Exit Pressure $\left(\mathrm{P}_{\mathrm{e}}\right) \quad=12.987$ bar

Ratio of Specific heats ( $\mathrm{Y}) \quad=1.4$

Gas Constant $(\mathrm{R}) \quad=287 \mathrm{~J} / \mathrm{KgK}$

According to CD Nozzle theory, the optimum Nozzle area ratio(NAR) for designing the nozzle is calculated by using the governing relations as calculated below

$$
\begin{aligned}
& \frac{T_{0}}{T^{*}}=1+\frac{Y-1}{2}\left(M^{*}\right)^{2} \\
& \frac{1583}{T^{*}}=1+\frac{1.4-1}{2}(1)^{2} \\
& T^{*}=1319,16 \mathrm{~K} \\
& a^{*}=\sqrt{Y R T^{*}} \\
& a^{*}=\sqrt{1.4 * 287 * 1319.16} \\
& a^{*}=728.04 \mathrm{~m} / \mathrm{s} \\
& \frac{P_{0}}{P^{*}}=\left\{1+\frac{Y-1}{2}\left(M^{*}\right)^{2}\right\}^{\frac{Y}{Y-1}} \\
& \frac{80}{P^{*}}=\left\{1+\frac{1.4-1}{2}(1)^{2}\right\}^{\frac{1.4}{1.4-1}} \\
& P^{*}=42.26 \text { bar } \\
& \frac{P_{\theta}}{P^{*}}=\left\{\frac{Y+1}{2+(Y-1)\left(M_{\theta}\right)^{2}}\right\}^{\frac{r}{Y-1}} \\
& \frac{12.987}{42.26}=\left\{\frac{1.4+1}{2+(1.4-1)\left(M_{e}\right)^{2}}\right\}^{\frac{1.4}{1.4-1}} \\
& M_{e}=1.845 \\
& \frac{T_{0}}{T_{\theta}}=1+\frac{Y-1}{2}\left(M_{\theta}\right)^{2} \\
& \frac{1583}{T_{e}}=1+\frac{1.4-1}{2}(1.845)^{2} \\
& T_{\theta}=941.81 \mathrm{~K} \\
& a_{e}=\sqrt{Y R T_{e}} \\
& a_{e}=\sqrt{1.4 * 287 * 941.81} \\
& a_{e}=615.157 \mathrm{~m} / \mathrm{s} \\
& \left(\frac{A_{e}}{A^{*}}\right)^{2}=\frac{1}{\left(M_{e}\right)^{2}}\left\{\frac{2}{Y+1}\left(1+\frac{Y-1}{2}(M)^{2}\right)\right\}^{\frac{Y+1}{\Gamma-1}} \\
& \left(\frac{A_{e}}{A^{*}}\right)^{2}=\frac{1}{(1.854)^{2}}\left\{\frac{2}{1.4+1}\left(1+\frac{1.4-1}{2}(1.854)^{2}\right)\right\}^{\frac{1.4+1}{1.4-1}}
\end{aligned}
$$


$\frac{A_{e}}{A^{*}}=1.489$

The above area ratio is the optimum Nozzle area ratio calculated from theoretical formulae for designing a nozzle for the taken input conditions. In the same way, the optimum area ratio is calculated for the gas properties which is found to be 1.594 .

The design considerations of the nozzle are taken from the literature study and calculated the optimum divergence angle that can be kept for the inlet flow conditions and properties taken. Throat area as per the design consideration is taken as 30.054 inches which is substituted in the area ratio to obtain the value of exit area. From the area ratio and the length of the divergent section, nozzle divergence angle is calculated as shown below

$$
\begin{aligned}
& \frac{A_{e}}{30.054}=1.489 \\
& A_{e}=44.75 i^{2} \\
& \alpha=2 \tan ^{-1}\left(\frac{\text { Radial length of divergent section }}{\text { Axial length of divergence section }}\right) \\
& \alpha=2 \tan ^{-1}\left(\frac{0.729}{17.055}\right) \\
& \alpha=5^{\circ}
\end{aligned}
$$

The favorable divergence angle is found to be $5^{0}$ as per the theoretical calculations done and the same is used to carry out the analysis work. There is no condition stating that this is the best divergence angle and it is regarded as the favorable value calculated for the taken input conditions. The theoretical calculations are based on the assumption that the flow is purely isentropic which is not valid in real cases. Hence, the divergence angle may deviate and the best angle of divergence is found by numerical simulation carried out using Ansys CFX.

The Analysis is carried out for different divergence angles of $5^{0}, 9^{0}, 11^{0}, 15^{0}$ and $20^{\circ}$ and the nozzle giving best performance is shown in the simulation work. It is also found that the results obtained analytically are in good agreement with theoretical relations used thus validating the results. The same analysis is done by taking an expansion gas and a comparison is made between the air and gas performance.

\section{Results and Discussions}

The Simulation is carried out for air and expansion gas the plots of which are shown and discussed in this section.

Of all the results obtained analytically, the objective is to show the best angle of divergence, Nozzle pressure ratio(NPR) and Nozzle area ratio(NAR) for which the nozzle is giving higher exit mach.

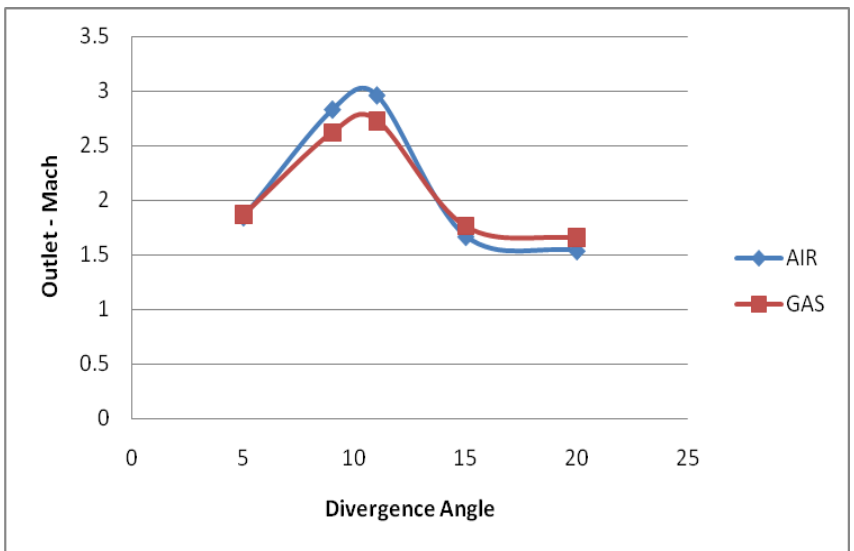

Chart-1: Outlet Mach vs. Divergence Angle

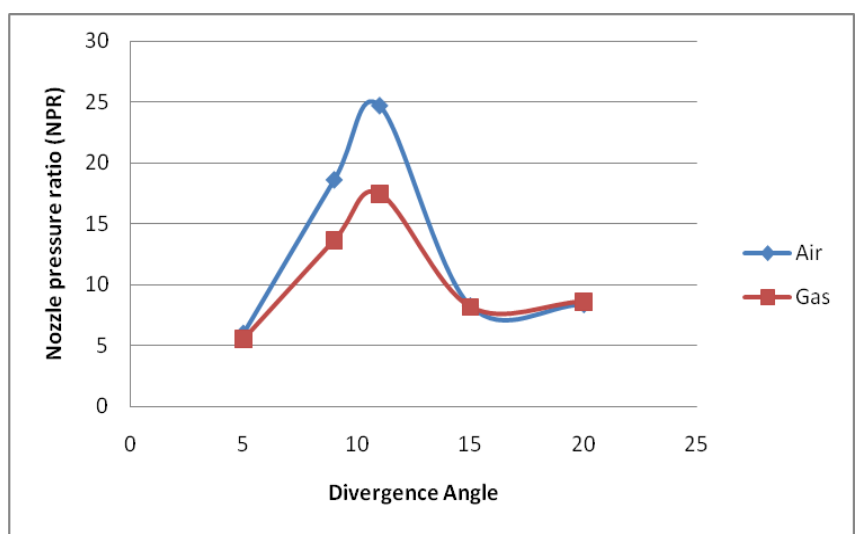

Chart-2: Nozzle pressure ratio vs. divergence angle

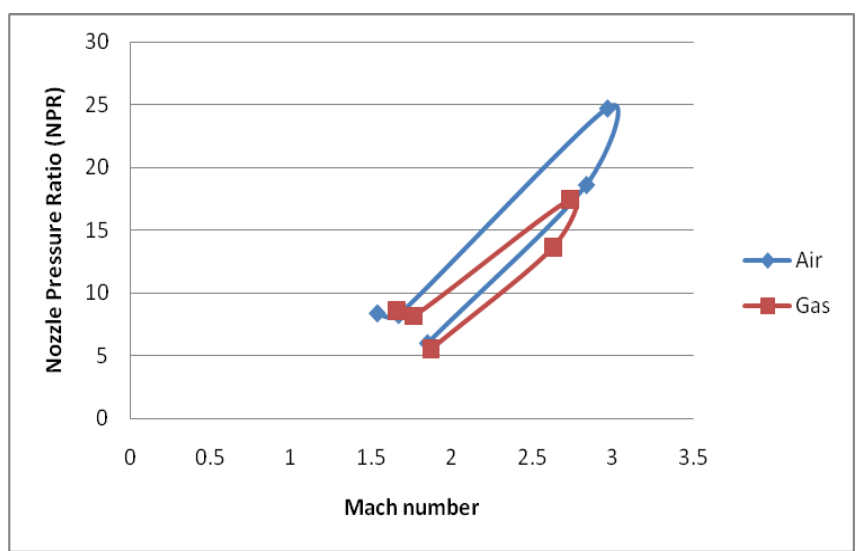

Chart-3: Mach number vs. Nozzle pressure ratio

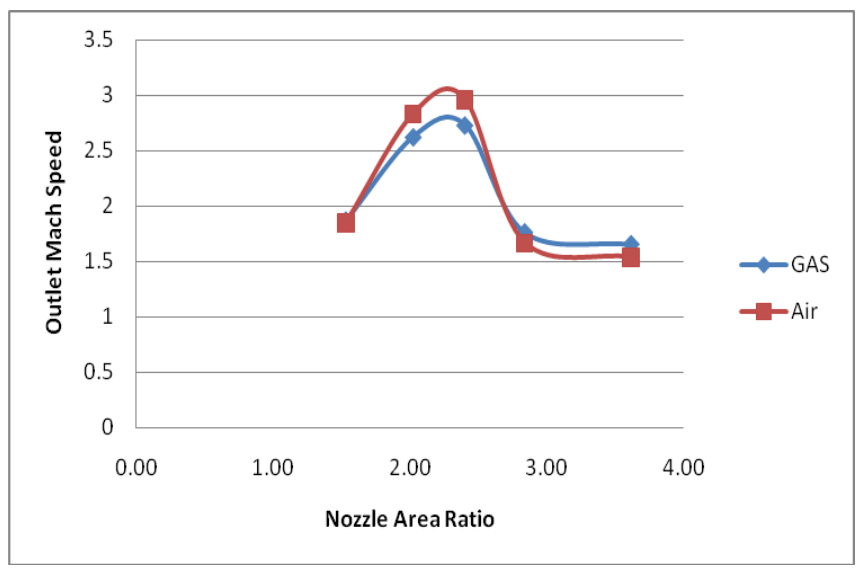

Chart-4: Outlet Mach vs. Nozzle Area Ratio (NAR) 
From the chart-1, it is clear that the nozzle with divergence angle of $11^{\circ}$ is giving the best performance in comparison with other nozzles for both air as well as expansion gas. It is also evident that the performance of air is higher in comparison with expansion gas upto $15^{\circ}$ angle of divergence. Crossing the angle of $15^{\circ}$ is resulting in good exit mach for expansion gas. The maximum exit mach is found to be 2.96 for air and 2.73 for gas. It is also in accordance with the theory that nozzle having higher pressure ratio gives best performance as shown in chart 2 . The best Nozzle pressure ratios(NPR's) obtained for air and gas are 24.72 and 17.46 respectively.

In the similar way it is found that same trend is followed for nozzle area ratio(NAR). The best NAR for air as well as gas is found to be 2.4 as the angle of divergence is same for best exit mach.

\section{CONCLUSIONS}

$>$ For the taken input flow conditions, the optimum area ratio is calculated for air and gas which are found to be 1.489 and 1.594 .

$>$ The results obtained analytically are in accordance with theoretical relations used thus validating the results.

$>\quad$ The best nozzle performance is obtained for nozzle with $11^{\circ}$ angle of divergence for both air and gas. So the CD nozzle of $11^{0}$ is the best nozzle to accelerate the flows upto supersonic mach speeds. These can be used as steam nozzles in steam turbines.

$>\quad$ The maximum exit mach obtained for air is 2.96 and 2.73 for expansion gas. The air is found to perform better in low divergent angle CD nozzles.

$>\quad$ The best Nozzle Pressure Ratio (NPR) values are found to be 24.72 and 17.46 for air and expansion gas. However as the best nozzle performance obtained for air and gas is at same divergence angle, the best Nozzle Area Ratio(NAR) for air and gas is same which is found to be 2.4 .

\section{REFERENCES}

[1]. G.Satyanarayana, Ch.Varun and S.S.Naidu., "CFD analysis of a Convergent-Divergent nozzle", ACTA TECHNICA CORVINIENSIS - Bulletin of Engineering TOME VI (2013) fascicule 3 IISN 20673809

[2]. Omid Joneydi Shariatzadeh, Afshin Abrishamkar, and AliakbarJoneidi jafari "Computational Modeling of a Typical Supersonic Convergent-Divergent Nozzle and Validation by Real Measured Data"., JOCET 2015 Vol.3(3): 220-225,ISSN:1793-821X, DOI: 10.7763/ JOCET.2015. V3.198

[3]. Fred W.Steffen, H.George Krull and Ralph F.Schmiedlin., "Effect of Divergence angle on the internal performance characteristics of several conical convergent-divergent nozzles, National Advisory Committee for Aeronautics.

[4]. Ms. B.Krishna Prafulla, Dr. V. Chitti Babu and Sri P. Govinda Rao., " CFD analysis of Convergent -
Divergent supersonic nozzle", International Journal of computational engineering research $\mathrm{Vol} 3$ Issue 5

[5]. K.M.Pandey,Member IACSIT and A.P.Singh, "CFD Analysis of a Conical Nozzle for Mach 3 at various angles of Divergence with Fluent Software", International Journal of Chemical Engineering and Applications, Vol.1, No.2, August 2010, ISSN: 20100221.

[6]. MD. Safayet Hossain, Muhammad Ferdous Raiyan, Nahed Hassan Jony "Comparative Study of Supersonic Nozzles" IJRET: International Journal of Research in Engineering and Technology eISSN: 2319-1163 | pISSN: 2321-7308

[7]. E.M.S.Ekanayake, J.A. Gear and Y.Ding "Flow Simulation of a two Dimensional Rectangular Supersonic Convergent -Divergent Nozzle" ANZIAM J.51 (EAMC 2009)pp.C377-C392-2010

[8]. Muhammad Ahsan, "Numerical analysis of a friction factor for a fully developed turbulent flow using $\mathrm{k}-\varepsilon$ turbulence model with enhanced wall treatment" BENI-SUEF university journal of basic and applied science 2014 